\title{
Outcome of ventral fusion of two or three cervical vertebrae with a locking compression plate for the treatment of cervical stenotic myelopathy in eight horses
}

\author{
Kühnle, Christoph ; Fürst, Anton E ; Ranninger, Elisabeth ; Suárez Sánchez-Andrade, José ; Kümmerle, Jan M
}

\begin{abstract}
Objectives: It was recently shown that biomechanical stability achieved with a locking compression plate (LCP) for ventral cervical fusion in horses is similar to the commonly used Kerf cut cylinder. The advantages of the LCP system render it an interesting implant for this indication. The goal of this report was to describe surgical technique, complications and outcome of horses that underwent ventral fusion of two or three cervical vertebrae with an LCP. Methods: Medical records of eight horses were reviewed for patient data, history, preoperative grade of ataxia, diagnostic imaging, surgical technique and complications. Follow-up information was obtained including clinical re-examination and radiographs whenever possible. Results: Two $(n=5)$ or $3(n=3)$ cervical vertebrae were fused in a mixed population with a median age of 9 months, median weight of $330 \mathrm{~kg}$ and median grade of ataxia of 3/5. A narrow 4.5/5.0 LCP $(\mathrm{n}=6)$, a broad 4.5/5.0 LCP $(\mathrm{n}=1)$ and a human femur 4.5/5.0 LCP $(\mathrm{n}=1)$ were applied. Two horses were re-operated due to implant loosening. Six patients developed a seroma. Long-term complications included ventral screw migration in four, spinal cord injury in one and plate breakage in two horses at 720 to 1116 days after surgery. Outcome was excellent in three, good in four, poor in one patient. Clinical Significance: The use of an LCP for ventral cervical vertebral fusion is associated with good clinical results. However, a careful surgical technique is required to further reduce the complication rate.
\end{abstract}

DOI: https://doi.org/10.1055/s-0038-1666979

Posted at the Zurich Open Repository and Archive, University of Zurich ZORA URL: https://doi.org/10.5167/uzh-160742

Journal Article

Published Version

Originally published at:

Kühnle, Christoph; Fürst, Anton E; Ranninger, Elisabeth; Suárez Sánchez-Andrade, José; Kümmerle, Jan M (2018). Outcome of ventral fusion of two or three cervical vertebrae with a locking compression plate for the treatment of cervical stenotic myelopathy in eight horses. Veterinary and Comparative Orthopaedics and Traumatology, 31(5):356-363.

DOI: https://doi.org/10.1055/s-0038-1666979 


\title{
Outcome of Ventral Fusion of Two or Three Cervical Vertebrae with a Locking Compression Plate for the Treatment of Cervical Stenotic Myelopathy in Eight Horses
}

\author{
Christoph Kühnle ${ }^{1}$ Anton E. Fürst ${ }^{1}$ Elisabeth Ranninger ${ }^{2}$ José Suárez Sánchez-Andrade ${ }^{3}$ \\ Jan M. Kümmerle ${ }^{1}$
}
1 Division of Equine Surgery, Equine Hospital, Equine Department, Vetsuisse Faculty, University of Zurich, Zurich, Switzerland
${ }^{2}$ Equine Hospital, Division of Anaesthesiology, Equine Department, Vetsuisse Faculty, University of Zurich, Zurich, Switzerland
${ }^{3}$ Small Animal Hospital, Division of Diagnostic Imaging, Small Animal Department, Vetsuisse Faculty, University of Zurich, Zurich, Switzerland

Vet Comp Orthop Traumatol 2018;31:356-363.

\begin{abstract}
Keywords

- Wobbler syndrome

- equine surgery

- cervical fusion

- locking plates

- spine surgery

Objectives It was recently shown that biomechanical stability achieved with a locking compression plate (LCP) for ventral cervical fusion in horses is similar to the commonly used Kerf cut cylinder. The advantages of the LCP system render it an interesting implant for this indication. The goal of this report was to describe surgical technique, complications and outcome of horses that underwent ventral fusion of two or three cervical vertebrae with an LCP.

Methods Medical records of eight horses were reviewed for patient data, history, preoperative grade of ataxia, diagnostic imaging, surgical technique and complications. Follow-up information was obtained including clinical re-examination and radiographs whenever possible.

Results Two $(n=5)$ or $3(n=3)$ cervical vertebrae were fused in a mixed population with a median age of 9 months, median weight of $330 \mathrm{~kg}$ and median grade of ataxia of 3/5. A narrow 4.5/5.0 LCP $(n=6)$, a broad 4.5/5.0 LCP $(n=1)$ and a human femur 4.5/ 5.0 LCP $(n=1)$ were applied. Two horses were re-operated due to implant loosening. Six patients developed a seroma. Long-term complications included ventral screw migration in four, spinal cord injury in one and plate breakage in two horses at 720 to 1116 days after surgery. Outcome was excellent in three, good in four, poor in one patient.

Clinical Significance The use of an LCP for ventral cervical vertebral fusion is associated with good clinical results. However, a careful surgical technique is required to further reduce the complication rate.
\end{abstract}

received

November 21, 2017

accepted after revision

May 9, 2018 (c) 2018 Georg Thieme Verlag KG Stuttgart · New York
DOI https://doi.org/

10.1055/s-0038-1666979.

ISSN 0932-0814. 


\section{Introduction}

Cervical vertebral stenotic myelopathy (CVSM), also known as equine wobbler syndrome, is a common cause of ataxia in young horses. ${ }^{1}$ Young male horses are usually overrepresented within the patient population. ${ }^{1-3}$ Although CVSM is described in almost all horse breeds, Thoroughbreds, Warmbloods and Tennessee Walking Horses appear to be more frequently affected. ${ }^{1,4}$

The diagnosis requires a detailed patient history, physical and neurological examination as well as diagnostic imaging. ${ }^{5}$ Evaluation of plain radiographs in combination with calculation of the intra- and intervertebral minimal sagittal ratios or diameter can initially raise a valid suspicion of CVSM. ${ }^{2,6}$ However, the risk of a false-positive diagnosis made with plain radiographs can be up to $50 \%{ }^{3}$ Therefore, for definitive CVSM diagnosis a myelographic examination should be performed to confirm the findings ${ }^{3}$ and the exact location of the stenosis-in particular if surgical treatment is intended. ${ }^{6}$ Myelography allows for classification into type I (dynamic) and type II (static) CVSM. Dynamic compressive lesions impinge on the spinal cord depending on cervical position, typically only visible in the flexed cervical spine. Static compressive lesions impinge on the cervical spine regardless of cervical position. Type I (dynamic) lesions are most commonly found in the mid-cervical area while type II (static) lesions occur most frequently in the caudal part of the cervical spine. ${ }^{7}$ Dynamic lesions are observed more frequently than static lesions. ${ }^{4}$ Specifically, spinal cord compression occurs most frequently at cervical vertebrae (CV) 3 and $4(18-32 \%)^{8}$ followed by CV 6 to $7(17 \%)$ and CV 5 to 6 (14\%). ${ }^{3}$ Furthermore, multiple compressive lesions were found in $29 \%$ of horses diagnosed with CVSM in one study. ${ }^{3}$ Similarly, another recent study found single-level stenosis in 37\%, double-level stenosis in $35 \%$ and triple-level stenosis in $13 \%$ of their patients examined myelographically during the diagnostic work-up of suspected CVSM. ${ }^{4}$ The most frequent combination of double-level stenosis is CV 3 to 4 and CV 5 to 6 followed by CV 3 to 4 and $\mathrm{CV} 4$ to 5 . $^{3}$

Additionally, recent studies emphasize the benefits of advanced imaging techniques such as computed tomography (CT) or magnetic resonance imaging over plain radiographs. ${ }^{9,10}$

Beside conservative treatment with restricted diet and activity, ${ }^{2}$ the recommended surgical therapy for the treatment of CVSM in horses is ventral cervical vertebral fusion. For this purpose, the kerf cut cylinder (KCC) is most commonly used. ${ }^{11}$ The KCC used nowadays is a modification of the Cloward Bagby Basket, which was designed for vertebral fusion in human medicine. ${ }^{12}$ The titanium-based KCC, also known as 'Seattle Slew Implant,' can be fully or partially threaded, which is an important modification introduced to prevent implant migration. Special equipment is required for its implantation. The KCC is implanted between the affected vertebrae from the ventral aspect with the intervertebral disc centred in the middle of the open-ended cylinder. An isthmus of bone remains in the centre of the implant. Additionally, after partial removal of the intervertebral disc, the KCC is filled with a bone graft, which is re-vascularized through the open ends as well as little holes in the implant itself. In one study 126 horses were treated for CVSM with the KCC: $77 \%$ survived surgery without complications, $17 \%$ showed minor complications and $6 \%$ had fatal complications. ${ }^{13}$ Other studies in which the KCC was not exclusively used reported a wider range of success $(43-79 \%)^{7,13-17}$ and a fatal failure rate of up to $8 \%{ }^{7}$ However, in the most recent case series $57 \%$ of all horses that had ventral cervical fusionwhich was mainly done using the KCC technique-were euthanatized because of either vertebral body fractures or implant migration. ${ }^{4}$

Biomechanical in vitro studies indicated that locking compression plate (LCP) fixation results in similar or even superior biomechanical stability compared with KCC fixation for ventral cervical fusion of equine CV 4 and $5 .{ }^{18,19}$ Multiple site ventral cervical fusion was not evaluated in these biomechanical studies. ${ }^{18,19}$ The authors of these studies concluded that their results would justify the use of an LCP for ventral cervical fusion in clinical cases. ${ }^{18,19}$ However, there is hardly any literature that describes clinical experiences with use of an LCP technique for ventral cervical fusion of two or three $\mathrm{CV}$ in horses. ${ }^{20,21}$

The goal of this report was to describe surgical technique, complications and outcome in eight horses suffering from CVSM that underwent ventral fusion of two or three CV with an LCP at our institution.

\section{Materials and Methods}

All horses that had ventral cervical fusion with an LCP for the treatment of CVSM at our institution were included. Medical records from 2005 to 2015 were used to retrieve information on age, sex, breed, body weight, presentation, history, preoperative grade of ataxia, diagnostic imaging, surgical technique, anaesthesia length, recovery from anaesthesia, postoperative complications, postoperative treatment and outcome. Ataxia was graded from 0 to 5.22

Cervical vertebral stenotic myelopathy was diagnosed based on the results of clinical and neurological examinations as well as diagnostic imaging including plain radiographs obtained standing and myelography (Ultravist-370; Bayer AG, Zurich, Switzerland) CT (Siemens Somatom Sensation 40-slice spiral CT; Siemens, Zurich, Switzerland) and CT myelography under general anaesthesia. In addition, plain radiographs were repeated immediately before myelography. The intervertebral sagittal ratio was measured before surgery. ${ }^{6}$ All images were evaluated by ECVS and ECVDI diplomats.

\section{Surgical Technique}

All horses were pre-medicated with systemic antibiotic medications and non-steroidal anti-inflammatory drugs. The horses were sedated with medetomidine (Dorbene ad us. vet.; Dr. E. Graeub AG, Bern, Switzerland) (7 $\mu \mathrm{g} / \mathrm{kg}$, IV), and then induced with a mixture of ketamine (Ketanarkon 100 ad us. vet.; Streuli Pharma AG, Uznach, Switzerland) $(2.2 \mathrm{mg} / \mathrm{kg}$, IV) and diazepam (Valium; Roche Pharma AG, Reinach, Switzerland) $(0.02 \mathrm{mg} / \mathrm{kg}$, IV) and placed in dorsal recumbency with the neck positioned as straight as possible. 
Anaesthesia was maintained with isoflurane (Isoflurane ad us. vet.; Provet AG, Lyssach, Switzerland) in oxygen and a medetomidine (Dorbene ad us. vet.) constant rate infusion $(3.5 \mu \mathrm{g} / \mathrm{kg} / \mathrm{h}, \mathrm{IV})$. The correct position of the neck and especially the CV was ensured with padding, air filled bags and controlled after and using fluoroscopy (Siremobil C3D; Siemens Medical Solutions, Erlangen, Germany). The ventral aspect of the neck was clipped and prepared aseptically. A standard surgical ventral approach to the affected CV was performed. ${ }^{11}$ The ventral spine of the $\mathrm{CV}$ was flattened using an oscillating saw or osteotome. The intervertebral disc was partially removed using a Ruskin Rongeur, spoon curette or 3.2 to $4.5 \mathrm{~mm}$ drills depending on surgeon's preference.

\section{Plate Application}

A stainless-steel LCP (Synthes; Oberdorf, Switzerland) was applied to the ventral surface of the affected vertebrae under fluoroscopic control. Length and type of the plate depended on the location and extent of the cervical instability. The plate was carefully contoured to the surface of the vertebrae. Whenever possible, $5.0-\mathrm{mm}$ locking head screws (Synthes) were inserted into the plate holes; 4.5 - or $5.5-\mathrm{mm}$ cortical screws (Synthes) were only used if angulation of a screw was required or the threaded part of the combi-hole was damaged. All plate holes were filled with screws and no screws were placed in load position or in lag fashion. Under repeated fluoroscopic control screws were placed as close as possible to the spinal canal to maximize screw length and bone purchase. Synthetic absorbable suture material was used to appose the longus colli muscles, the remaining ventral cervical musculature and the subcutaneous tissue. In the minority of the horses an active drain (Blake; Ethicon Inc., Somerville, New Jersey, United States) was applied. The skin was closed with skin staplers and a sterile stent bandage was applied over the incision. All horses received morphine (Morphin HCL Sintetica; Sintetica S.A., Mendrisio, Switzerland) $(0.1 \mathrm{mg} / \mathrm{kg}$, IM) 30 minutes before being placed in the recovery stall.

\section{Postoperative Care}

Assistance for recovery from general anaesthesia was performed using either head and tail rope support or direct hand assistance by experienced technicians. All horses received medetomidine (Dorbene ad us. vet.) ( $2 \mu \mathrm{g} / \mathrm{kg}$, IV) for recovery as well as postoperative systemic antimicrobial medication and nonsteroidal anti-inflammatory drugs (NSAID) for at least 10 days postoperatively. In cases where an active drain (Blake) system was implanted in surgery, this drain was maintained until a reduction in wound secretion was noted.

\section{Follow-up and Outcome}

Short-term complications were defined as complications observed during hospitalization, whereas all adverse events after hospital discharge were categorized as long-term complications. Follow-up information was obtained by clinical and radiographic re-examination at the hospital or at the farm and via telephone interviews with the owners.

Outcome was defined as:

- Excellent: No ataxia, or used at intended athletic level, or both

- Good: Ataxia improved at least 1 grade, or used for pleasure riding, or both

- Fair: Slight improvement in ataxia, or not used for riding, or both

- Failure: No improvement in ataxia and death or euthanasia due to ataxia.

\section{Results}

\section{Horses}

The eight horses were a mixed population between 3 and 24 months of age (median: 9 months) and a median bodyweight of $330 \mathrm{~kg}$ (range: $200-490 \mathrm{~kg}$ ). Age, sex, breed and weight are summarized in - Table $\mathbf{1}$. Seven out of the eight horses were handled but not trained at the time of referral. One horse was in training and used as a trotter before it was presented. Two (No. 4 and 5) horses were discharged after the first presentation for conservative therapy before being represented (47 and 236 days later) for surgery.

\section{History}

None of the horses had ataxia related to an obvious trauma nor did the owner observe any episode of trauma. However, three horses were found in the paddock with a sudden onset

Table 1 Patient data, duration of symptoms and grade of ataxia at admission

\begin{tabular}{|l|l|l|l|l|l|l|}
\hline Case No. & Age (month) & Body weight $(\mathbf{k g})$ & Sex & Breed & Duration of symptoms & Grade of ataxia \\
\hline 1 & 11 & 340 & M & PRE & 2 days & 2 \\
\hline 2 & 3 & 200 & F & WB & 9 weeks & 3 \\
\hline 3 & 24 & 490 & M & STB & 17 weeks & 3 \\
\hline 4 & 21 & 360 & M & WB & 1 week & 3 \\
\hline 5 & 12 & 350 & F & WB & 30 weeks & 3 \\
\hline 6 & 6 & 290 & M & WB & 2 days & 3 \\
\hline 7 & 7 & 320 & M & FB & 5 weeks & 3 \\
\hline 8 & 7 & 260 & F & STB & 4 weeks & 3 \\
\hline
\end{tabular}

Abbreviations: F, female; FB, Freiberger; M, male; PRE, Pura raza española; STB, Standardbred; WB, Warmblood. Note: Grade of ataxia defined in the text. 
of ataxia and three horses had a previous growth spurt. The reported duration of ataxia before presentation for surgery was between 2 days and 30 weeks (median 35 days). Clinical signs were consistent with cervical spinal ataxia in all patients. The median ataxia grade was $3 / 5 .^{22}$

\section{Diagnostic Imaging}

Plain radiographs were taken in all eight horses. In one case (No.1) subluxation of CV to 2 was obvious. Radiographic findings consistent with deformation of the CV typical of CVSM were present in all horses. These included flare of the caudal epiphysis of the vertebral body, subluxation of adjacent vertebrae, abnormal ossification of the articular processes, osteoarthritis of the articular processes and extension of the dorsal lamina. ${ }^{5}$ Narrowing of the vertebral canal diameter defined as decreased intervertebral sagittal ratio ${ }^{5,6}$ was diagnosed in six of seven patients.

Myelography (Ultravist-370) was performed in seven horses and revealed dynamic extradural spinal cord compression at CV 3 to $4(n=2)$ and CV 4 to $5(n=3)$ and at CV 6 to 7 $(n=2)$. In all cases the contrast column was compressed more than $50 \%$ dorsally and ventrally in flexed position for CV 3 to 5 and in neutral position for CV 6 to 7 at the site of the CVSM allowing a diagnosis with adequate sensitivity and specifity. ${ }^{8} \mathrm{~A}$ standard CT (Siemens Somatom Sensation 40-slice spiral CT) study was performed in two patients (No. 1 and 8) and confirmed the spinal cord compression in both. A CT (Siemens Somatom Sensation 40-slice spiral CT) myelography (Ultravist-370) revealed a fragment in the cranial cervical articular facet of the fourth CV in one horse (No. 8). In horse No.1 myelography (Ultravist-370) was not performed because subluxation of CV 1 to 2 was obvious on plain radiographs and CT (Siemens Somatom Sensation 40-slice spiral CT) images.

\section{Surgery}

Ventral fusion under fluoroscopic (Siremobil C3D) control involved three CV in three horses and two vertebrae in five horses (-Table 2). Part of the ventral crista of the cranial affected vertebrae was removed in seven cases. Partial removal of the intervertebral disc was performed in all cases except one (No. 1). The bone that was removed from the

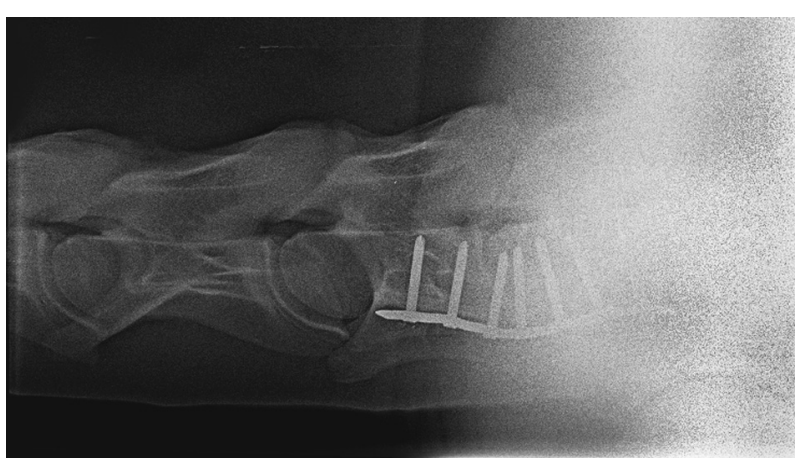

Fig. 1 Lateral radiograph of the sixth and seventh cervical vertebrae of case No. 3 obtained 4 days after surgery. A 7-hole narrow 4.5/5.0 locking compression plate with seven self-tapping 5.0-mm locking screws was placed.

ventral crista was used as a bone graft and placed in the ventral intervertebral space in three cases (No. 2, 4 and 8 ). ${ }^{23}$ A narrow 4.5/5.0 LCP (Synthes) was contoured to the ventral aspect of the $\mathrm{CV}$ in most cases (-Table 2). Exceptions were case No. 1 that had a human distal femur 4.5/5.0 LCP (Synthes) applied to fuse CV 1 to 2 and case No. 3 in which a broad 4.5/5.0 LCP (Synthes) was used to fuse CV 6 to 7 (-Fig. 1). Plate length for fusion of $3 \mathrm{CV}$ was 13 to 14 holes. For fusion of $2 \mathrm{CV}$, seven to nine-hole plates were implanted with the exception of case No. 1 that had the 5-hole distal femur LCP applied.

Plate contouring was classified as mild in three (No. 1, 2 and 4), moderate in three (No. 3, 5 and 8) and pronounced in two cases (No. 6 and 7). Most plate holes were filled with 5.0 locking screws (Synthes). Self-drilling locking screws were used in one patient (No. 2). On average, $85 \%$ of all screws were locking head screws; the 4.5 - or 5.5 -mm cortical screws were inserted in six patients (No. 1, 4, 5, 6, 7 and 8). An active Blake suction drain (Blake; Ethicon Inc., Somerville, New Jersey, United States) was placed before closure in three patients (No. 1, 3 and 7).

Surgery time ranged from 120 to 270 minutes (median: 178 minutes; mean: 186 minutes). Recovery from general anaesthesia was without major complications.

Table 2 Specification of implants and outcome

\begin{tabular}{|l|l|l|l|l|l|}
\hline Case No. & Locking compression plate & Fused vertebrae & Ataxia long-term & Use & Outcome $^{\mathbf{b}}$ \\
\hline 1 & 5-hole 4.5/5.0 femur & CV 1-2 & 2 & Pleasure horse & Good \\
\hline 2 & 7-hole 4.5/5.0 broad & CV 6-7 & 2 & Broodmare & Good \\
\hline 3 & 7-hole 4.5/5.0 narrow & CV 6-7 & 0 & Racehorse & Excellent \\
\hline 4 & 14-hole 4.5/5.0 narrow & CV 3-5 & 0 & Sport horse & Excellent \\
\hline 5 & 9-hole 4.5/5.0 narrow & CV 3-4 & 4 & Euthanatized & Poor \\
\hline 6 & 13-hole 4.5/5.0 narrow & CV 3-5 & 2 & Yearling & Good \\
\hline 7 & 13-hole 4.5/5.0 narrow & CV 3-5 & 0 & Sport horse & Excellent \\
\hline 8 & 8-hole 4.5/5.0 narrow & CV 3-4 & 2 & Yearling & Good \\
\hline
\end{tabular}

Abbreviation: CV, cervical vertebra.

${ }^{a} G$ rade of ataxia is defined in the text.

${ }^{b}$ Outcome is defined in the text. 


\section{Postoperative Medication}

Five horses were administered cefquinome (Cobactan IV 4.5\% ad us. vet; MSD Animal Health GmbH, Lucerne, Switzerland) for a mean time of 11 days (range: 3-16 days) and three horses received a combination of sodium penicillin G (Penicillin Natrium Streuli ad us. vet.; Streuli Pharma AG, Uznach, Switzerland) and gentamicin (Genta $100 \mathrm{mg} / \mathrm{mL}$; ad us. vet; CP-Pharma Handelsgesellschaft $\mathrm{mbH}$, Burgdorf, Germany) for a mean time of 7 days (range: 3-13 days). The mean administration of NSAID was 21 days (range: 10-40 days).

\section{Intraoperative Complications}

In case No. 1 the drill bit penetrated the spinal canal without sliding into it, causing mild bleeding. However, no other complication was observed that could be attributed to this event. In two (No. 1 and 7) cases the threads of four locking head screws were not engaging the bone when being delivered and were replaced by 5.5-mm cortex screws. In case No. 1 two locking head screws were replaced in the second surgery after the initial screws had been pulled out of the bone.

\section{Short-Term Complications}

Two horses (No. 1 and 2) were re-operated within 12 days (range: 1-12 days) due to ventral screw migration and implant loosening. In case No. 1 the initially placed screws were too short, which resulted in partial implant pull out one day after the first surgery. This horse had all screws replaced by longer implants in the second surgery. In the other case self-drilling screws had been implanted but became progressively loose over a 12-day period. At re-operation, all self-drilling locking screws (Synthes) were replaced by standard self-tapping locking screws (Synthes).

Five horses developed a seroma after the first surgery (No. $2,4,5,6$ and 8 ) and one horse (No. 1) after the second surgery. Five seromas were opened for drainage (No. 1, 2, 4, 6 and 8). After the routine pre- and postoperative antibiotic medication, four horses (No. 1, 4, 5 and 8) had clinical signs consistent with surgical site infection and were treated with marbofloxacin (Marbocyl 10\% ad us. vet, Vétoquinol AG, Bern, Switzerland) for a median of 45 days (range: $7-$ 104 days) and 1 horse (No.7) with doxycycline (Primodox 50 ad us. vet, ufamed AG, Sursee, Switzerland) for 8 days.

\section{Long-Term Complications}

Screw loosening and ventral screw migration after hospital discharge occurred in four horses (No. 1, 3, 4 and 7). Loosening affected all locking screws (Synthes) within one vertebra in one case (No. 7). Dorsal implant migration into the vertebral canal occurred secondary to insufficient plate contouring with a gap between the cranial end of the plate and the bone in another patient (No. 5) and was diagnosed 215 days after discharge. This horse was euthanatized because of progressive ataxia due to the spinal cord damage caused by the migrating screws (-Fig. 2). Plate breakage occurred at both intervertebral locations in two horses (No. 4 and 7 ) that had three vertebrae fused (-Fig. 3). In one of these horses (No. 7) pronounced plate contouring had been

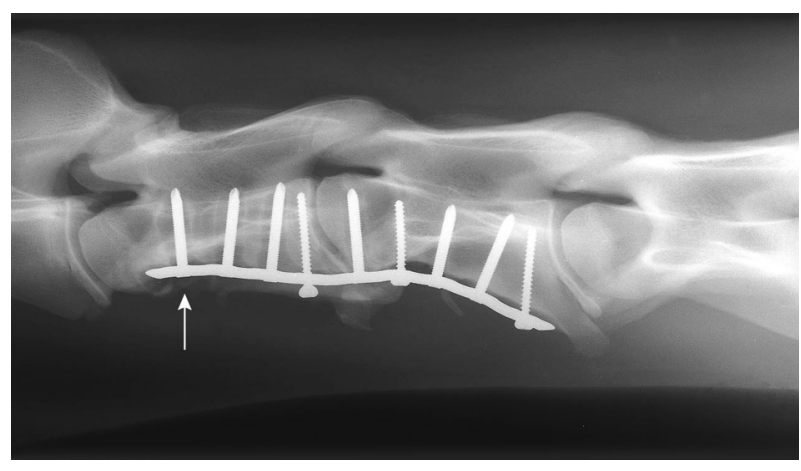

Fig. 2 Lateral radiograph of the second to fifth cervical vertebrae of patient No. 5, 7 months after surgery. Cranial is on the left of the image. The most cranial screws had entered the spinal canal. Insufficient plate contouring left a gap between bone and plate. This resulted in dorsal migration of the platelocked screw unit (white arrow).

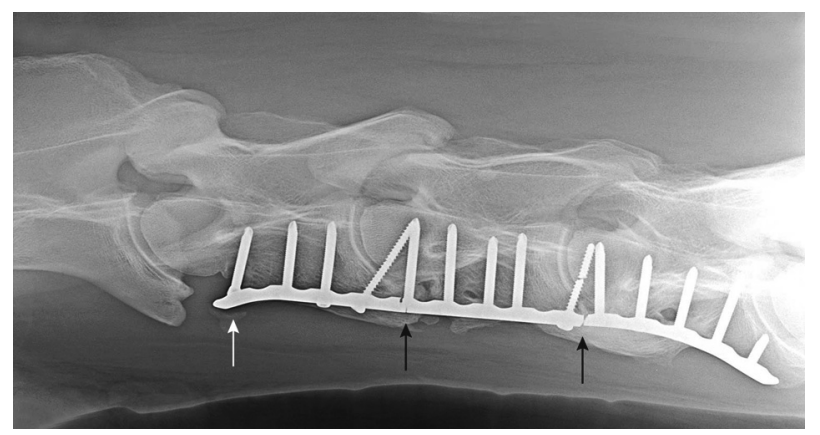

Fig. 3 Lateral radiograph of the second to fifth cervical vertebrae of patient No. 4 obtained almost 5 years after surgery. Cranial is on the left of the image. The 14-hole narrow 4.5/5 locking compression plate is broken between C3-4 and C4-5 (black arrows) and the most cranial locking screw is pulled out of the plate (white arrow). Despite the failed implant, the horse improved clinically and is used at intended athletic level.

performed in surgery. Breakage in both cases was first noticed on radiographs 710 (No. 7) and 857 (No. 4) days after surgery. In another case (No. 2) a broken locking screw (Synthes) was evident radiographically 3669 days postoperatively. Furthermore, follow-up radiographs of case No. 4 revealed that the most cranial locking screw (Synthes) was pulled out of the plate (-Fig. $\mathbf{3}$ ).

\section{Follow-up and Outcome}

Median follow-up was 482 days with a range of 84 to 3669 days. For seven patients, radiographs and results of a neurological examination performed by a veterinarian or videos for evaluation were available. In one case (No. 3 ) the follow-up examination included only a neurological examination and a video showing the horse in training.

Outcome was excellent in three patients (No. 3, 4 and 7), good in 4 (No. 1, 2, 6 and 8) and poor in 1 (No. 5) patient. Marked new bone formation bridging the ventral aspect of the fused vertebra was evident in all patients with radiographs available at least 4 months postoperatively (-Fig. 4). The newly formed bone was only present at the ventral aspect of the vertebrae (-Fig. 4) but hardly in the intervertebral space. 


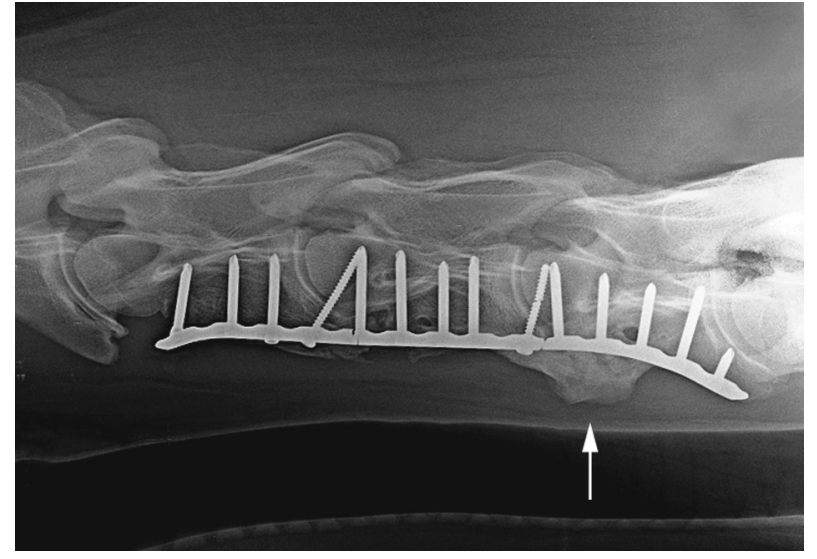

Fig. 4 Lateral radiograph of the second to fifth cervical vertebrae of patient No. 4 obtained more than 2 years after surgery. Cranial is on the left of the image. Marked ventral bone bridging is shown between the fourth and fifth cervical vertebrae (white arrow). However, bridging in the intervertebral space is hardly visible.

\section{Discussion}

This study describes a series of patients treated with an LCP for ventral cervical vertebral fusion and includes radiographic as well as clinical long-term follow-up examination. The clinical long-term outcome achieved with this technique compares favourably to the results reported with the KCC technique.

A high rate of seroma formation was observed, similar to other recent reports. ${ }^{21}$ Possibly, the more extensive approach required for plate insertion compared with the KCC technique increases the risk of seroma formation. The use of an active draining system prevented seroma formation in two out of three cases. In the single case where the active drain did not fully prevent seroma formation after the second surgery the seroma was small enough that it resolved without drainage. Seroma formation in combination with clinical signs of infection resulted in the prolonged use of antibiotic medications and increased hospitalization time in several patients. It might also contribute to subsequent problems such as implant loosening.

Complications such as dorsal migration of the platelocked screw unit and implant loosening were related to technical errors such as submaximal screw length, reduced thread length of self-drilling screws or insufficient plate contouring with gap formation between the plate and bone. However, pronounced contouring of the plate weakens it and makes breakage more likely. ${ }^{24}$ Conversely, performing marked ventral cervical body flattening results in removal of dense cortical bone. This decreases the screws purchase and screw length and makes implant pull out more likely.

Ventral screw migration was common in this technique and has been reported before. ${ }^{21}$ However, in this study it appears that ventral screw migration and radiographically evident mildto-moderate lucent areas around the screws have no relevant effect on the clinical outcome. Additionally, a report about the older stainless steel basket implanted for ventral cervical fusion in horses using the Cloward technique stated that backing out of the cylinder by up to $50 \%$ still leads to osseous fusion and stability. ${ }^{15}$ This could be comparable to partial ventral screw migration and plate loosening. In this context it should be considered that a loose implant might not lead to complete osseous fusion, but at least to fibrous fusion. Fibrous fusion is not as stable as osseous fusion although it seems stable enough to reduce cord compression and therefore improving ataxia and clinical appearance. ${ }^{25}$ Whether the intraoperative application of polymethylmethacrylate (PMMA) on the screw heads could decrease the occurrence of ventral screw migration is undetermined and needs to be assessed. Given the higher rate of surgical site infection in this study, PMMA implantation could increase the risk for infection unless it is impregnated with antimicrobials. ${ }^{26}$ Additionally, PMMA application could make replacement of screws more difficult. A case series of 126 horses treated with the KCC technique found that ventral migration was observed in only $10 \%$ of patients. However, only a ventral displacement of the implant by more than $1 \mathrm{~cm}$ was considered as ventral implant migration. ${ }^{13}$ Ventral migration of a few screws in an LCP construct is most likely not as detrimental to the overall stability as ventral migration of the cylinder in the KCC technique.

Although the LCP technique performed by Reardon and colleagues $^{18,19}$ appears similar to the technique used in this study, there were some differences in the material and in the surgical technique. In this study we mainly used 4.5/5.0 narrow LCP instead of $4.5 / 5.0$ broad LCP, due to a better handling and placement. Furthermore, all plate holes were filled with screws, whereas the centre holes were left empty in the biomechanical study. Whether the construct is stronger with all holes filled remains unclear and needs further investigation. In equine osteosynthesis all plate holes are usually filled with screws. Screws placed in the mid-part of the plate entering both CV might enhance stiffness as well. On the other hand, leaving the mid-part without screws follows the concept of bridge plating, which distributes the deformation of the plate over a longer distance and reduces the strain. ${ }^{27}$

New bone formation bridging the intervertebral space was restricted to the ventral aspect of the fused vertebrae. Suboptimal osseous fusion exposes the implants to permanent cyclic stress and might contribute to breaking of the implant as noted in two horses with fusion of three vertebrae. Therefore, a broad LCP should be considered for fusion of three vertebrae to increase the area moment of inertia and thus bending stiffness. ${ }^{28}$ Currently, it remains unclear whether the use of a broad plate for multiple site fusion improves biomechanical implant performance sufficiently or if the KCC technique is advantageous in cases that require fusion at more than one location. Aggressive intervertebral disc removal, possibly in combination with application of an autogenous bone graft, could enhance osseous fusion and therefore relieve load on the implants. In this study a cortical bone autograft was used. It is unclear if use of a cancellous bone autograft would result in more new bone formation. An in vitro study on equine bone revealed that cortical bone specimens were associated with significantly more bone nodule formation from osteoprogenitor cells compared with cancellous bone specimens. ${ }^{29}$ A human periodontal study found no difference in the percentage of new bone 
formation between patients that had a cancellous versus a cortical bone graft. ${ }^{23}$

This retrospective case series has several limitations. Some of them, such as the small and heterogeneous patient population, use of different implants and a surgical technique adapting over time were associated with the retrospective nature of this study. A successful outcome probably depends on the number and location of fused vertebrae. Additionally, with eight cases in a 10 -year period, this surgical technique cannot be termed a routine procedure and contributed to the long surgery times described in this study compared with those reported for the KCC technique. The LCP application requires more dissection and technical steps than the KCC technique, also resulting in an increased surgery time. This along with the frequent seroma formation and surgical site infection is another disadvantage of the LCP technique. Finally, the duration of antibiotic therapy in case of surgical site infection was very long with a median of 45 days. Prolonged systemic administration of antimicrobials with a reported median duration of 21.8 days is commonly performed in case of orthopaedic infections in equine long bone fractures and arthrodeses treated by internal fixation. ${ }^{30}$ The protracted therapy with antibiotic medications in this study is difficult to justify without culture and sensitivity data and might promote the development of antimicrobial resistance.

In conclusion, LCP ventral cervical vertebral fusion is associated with good to excellent clinical results. Besides potential biomechanical advantages, the LCP technique has the advantage of easier availability than other systems. However, like every novel technique in early stages of development it needs to be adjusted to minimize complications.

\section{Conflict of Interest}

None.

\section{Author Contributions}

Christoph Kühnle, Anton E. Fürst and Jan M. Kümmerle contributed to conception of study, study design and acquisition of data and data analysis and interpretation. José Suárez Sánchez-Andrade contributed to acquisition of data and data analysis and interpretation. Elisabeth Ranninger contributed to acquisition of data. All authors drafted, revised and approved the submitted manuscript.

\section{References}

1 Levine JM, Ngheim PP, Levine GJ, Cohen ND. Associations of sex, breed, and age with cervical vertebral compressive myelopathy in horses: 811 cases (1974-2007). J Am Vet Med Assoc 2008;233 (09):1453-1458

2 Mayhew IG, Donawick WJ, Green SL, Galligan DT, Stanley EK, Osborne J. Diagnosis and prediction of cervical vertebral malformation in thoroughbred foals based on semi-quantitative radiographic indicators. Equine Vet J 1993;25(05):435-440

3 Papageorges M, Gavin PR, Sande RD, et al. Radiographic and myelographic examination of the cervical vertebral columns in 306 ataxic horses. Vet Radiol 1987;28:53-59

4 Szklarz M, Skalec A, Kirstein K, et al. Management of equine ataxia caused by cervical vertebral stenotic myelopathy: a European perspective 2010-2015. Equine Vet Educ 2018;30:370-376
5 Rush BR. Developmental vertebral anomalies. In: Auer JA, Stick JA, eds. Equine Surgery. 4th ed. Missouri: Elsevier Saunders; 2012: 693-700

6 Hahn CN, Handel I, Green SL, Bronsvoort MB, Mayhew IG. Assessment of the utility of using intra- and intervertebral minimum sagittal diameter ratios in the diagnosis of cervical vertebral malformation in horses. Vet Radiol Ultrasound 2008; 49(01):1-6

7 Moore BR, Reed SM, Robertson JT. Surgical treatment of cervical stenotic myelopathy in horses: 73 cases (1983-1992). J Am Vet Med Assoc 1993;203(01):108-112

8 van Biervliet J, Scrivani PV, Divers TJ, Erb HN, de Lahunta A, Nixon A. Evaluation of decision criteria for detection of spinal cord compression based on cervical myelography in horses: 38 cases (1981-2001). Equine Vet J 2004;36(01):14-20

9 Janes JG, Garrett KS, McQuerry KJ, et al. Comparison of magnetic resonance imaging with standing cervical radiographs for evaluation of vertebral canal stenosis in equine cervical stenotic myelopathy. Equine Vet J 2014;46(06):681-686

10 Kristoffersen M, Puchalski S, Skog S, et al. Cervical computed tomography (CT) and CT myelography in live horses: 16 cases. Equine Vet J 2014;46:11

11 Walmsley J, Grant BD. Surgical treatment of developmental diseases of the spinal column. In: Auer JA, Stick JA, eds. Equine Surgery. 4th ed. Missouri: Elsevier Saunders; 2012:700-711

12 McAfee PC. Interbody fusion cages in reconstructive operations on the spine. J Bone Joint Surg Am 1999;81(06):859-880

13 Schütte A. Untersuchungen zum equinen wobbler syndrom. (Examination of the equine wobbler syndrome). Doctoral thesis, Munich, Germany: Ludwig-Maximilians-Universität; 2005

14 Walmsley JP. Surgical treatment of cervical spinal cord compression in horses: a European experience. Equine Vet Educ 2005; 17:39-43

15 Grant BD, Barbee DD, Wagner PC, et al. Long term results of surgery for equine cervical vertebral malformation. Proc Am Assoc Equine Pract 1985;31:91-96

16 Nixon AJ, Stashak TS, et al. Surgical therapy for spinal cord disease in the horse. Proc Am Assoc Equine Pract 1985;31:61-74

17 Grant GB, Bagby G, Rantanen N, et al. Clinical results of kerf cylinder (Seattle Slew Implant) to reduce implant migration and fracture in horses undergoing surgical interbody fusion. Vet Surg 2003;32:499

18 Reardon R, Bailey R, Walmsley J, Heller J, Lischer C. A pilot in vitro biomechanical comparison of locking compression plate fixation and kerf-cut cylinder fixation for ventral fusion of fourth and fifth equine cervical vertebrae. Vet Comp Orthop Traumatol 2009;22 (05):371-379

19 Reardon RJ, Bailey R, Walmsley JP, Heller J, Lischer C. An in vitro biomechanical comparison of a locking compression plate fixation and kerf cut cylinder fixation for ventral arthrodesis of the fourth and the fifth equine cervical vertebrae. Vet Surg 2010;39 (08):980-990

20 Reardon R, Kummer M, Lischer C. Ventral locking compression plate for treatment of cervical stenotic myelopathy in a 3-monthold warmblood foal. Vet Surg 2009;38(04):537-542

21 Rossignol FV, Brandenberger O, Denoix JM. Use of locking compression plate fixation for ventral cervical arthrodesis to treat cervical instability in horse. ACVS Summit; Nashville, Tennessee; 2015

22 Mayhew IG, deLahunta A, Whitlock RH, Krook L, Tasker JB. Spinal cord disease in the horse. Cornell Vet 1978;68(Suppl 6):1-207

23 Eskow AJ, Mealey BL. Evaluation of healing following tooth extraction with ridge preservation using cortical versus cancellous freeze-dried bone allograft. J Periodontol 2014;85(04): 514-524

24 Fackelman GE, Bramlage LR, Auer JA, et al. AO principles of equine osteosynthesis: with AO teaching videos on CD-ROM. AO Pub; 2000:20 
25 DeBowes RM, Grant BD, Bagby GW, Gallina AM, Sande RD, Ratzlaff $\mathrm{MH}$. Cervical vertebral interbody fusion in the horse: a comparative study of bovine xenografts and autografts supported by stainless steel baskets. Am J Vet Res 1984;45(01):191-199

26 Ahern BJ, Richardson DW. Surgical site infection and the use of antimicrobials. In: Auer JA, Stick JA, eds. Equine Surgery. 4th ed. Missouri: Elsevier Saunders; 2012:68-84

27 Gautier E, Sommer C. Guidelines for the clinical application of the LCP. Injury 2003;34(Suppl 2):B63-B76
28 Muir P, Johnson KA, Markel MD. Area moment of inertia for comparison of implant cross-sectional geometry and bending stiffness. Vet Comp Orthop Traumatol 1995;8:146-152

29 McDuffee LA, Anderson GI, Wright GM, Ryan DA. In vitro heterogeneity of osteogenic cell populations at various equine skeletal sites. Can J Vet Res 2006;70(04):277-284

30 Ahern BJ, Richardson DW, Boston RC, Schaer TP. Orthopedic infections in equine long bone fractures and arthrodeses treated by internal fixation: 192 cases (1990-2006). Vet Surg 2010;39(05):588-593 